\title{
Which immunosuppressive drug is preferred in the treatment of toxic epidermal necrolysis during COVID-19 outbreak?
}

\author{
Saeedeh Farajzadeh ${ }^{1}$ and Najmeh Ahramiyanpour ${ }^{2}$ \\ ${ }^{1}$ Kerman University of Medical Sciences \\ ${ }^{2}$ Affiliation not available
}

February 23, 2021

\begin{abstract}
Toxic epidermal necrolysis (TEN) is a serious drug reaction. Its proper cure would be challenging during COVID-19 outbreak because of a dilemma regarding selecting the immunosuppressive drug. In this case presentation, we report a case of TEN who treat successfully with cyclosporine during COVID-19 outbreak in a referral COVID-19 hospital.
\end{abstract}

Title: Which immunosuppressive drug is preferred in the treatment of toxic epidermal necrolysis during COVID-19 outbreak?Authors: Saeedeh Farajzadeh ${ }^{1}$ M.D., Najmeh Ahramiyanpour ${ }^{1}$ M.D.Affiliations:

Department of Dermatology, Afzalipoor Hospital, Kerman University of Medical Sciences, Kerman, Iran

Corresponding Author: Najmeh Ahramiyanpour, M.D., Department of Dermatology, Afzalipoor Hospital, Kerman University of Medical Sciences, Kerman, Iran. Tel: +989173703643, E-mail: najmeh.pour@gmail.comAbstract: Toxic epidermal necrolysis (TEN) is a serious drug reaction. Its proper cure would be challenging especially during COVID-19 outbreak because of a dilemma regarding selecting the immunosuppressive drug. In this case presentation, we report a case of TEN who treat successfully with cyclosporine during COVID-19 outbreak in a referral COVID-19 hospital.Keywords: Toxic epidermal necrolysis, COVID-19, immunosuppressant, cyclosporine, calcineurin inhibitors.Introduction: Toxic epidermal necrolysis (TEN) is a rare drug reaction associated with high mortality rate ${ }^{[1]}$. TEN presents with erythematous and dusky maculopapular rash that progresses to flaccid blisters, epidermal erosion, necrosis and skin detachment. ${ }^{[1-3]}$ Drug hypersensitivity is the most common cause of TEN. TEN can be associated with infections, systemic diseases, malignancies and autoimmune conditions ${ }^{[4-6]}$. While there is no gold standard treatment for TEN, systemic corticosteroid, intravenous immunoglobulin (IVIG), cyclosporine, and tumor necrosis factor (TNF) inhibitors are optional drugs for the management of TEN ${ }^{[1-4]}$. Management of TEN is always challenging especially during COVID-19 outbreak because use of the immunosuppressive drugs might have both beneficial and harmful effects on COVID-19 course ${ }^{[7]}$. As we know there is not any study to compare the effects of different immunosuppressive drugs such as cyclosporine or prednisolone on TEN patients who are predispose to COVID-19. Selecting an immunosuppressive drug in a patient with TEN is challenging and troublesome. Here, we report a case of TEN who treat successfully with cyclosporine during COVID-19 outbreak in a referral corona hospital.Case Synopsis: A 24-year-old man came into the emergency department with a 4-days history of painful generalized dusky rash on his body associated mucosal surface involvement. His past medical history was positive for bipolar mood disorder. He was on carbamazepine and valproate sodium for the last two years and lamotrigine during the last two weeks. On arrival to the emergency room, the patient was oriented and his vital signs were stable (Bp: 130/80 $\mathrm{mmHg}$, pulse rate: 80 beats per minute, temperature: 36.9, respiratory rate: 16 per minute, and O2 saturation: $99 \%$ ). Physical examination described more than $50 \%$ of body surface area involvement. skin lesions were as painful, dusky and purpuric macules and patches of irregular size and shape on the trunk and extremities. 
Flaccid blisters with serosal fluid and detached epidermal surface on the neck and chest detected (Figure 1). The Nikolsky sign was positive. Hemorrhagic crust, lips erosion, erythema of buccal mucosa, conjunctivitis, and facial edema is seen He also complained of photophobia and painful dysphagia. His SCORTEN (SCORs of Toxic Epidermal Necrolysis) was 2. Laboratory tests revealed exclusively a mild leukopenia while other results were negative or in normal ranges: potassium: $4 \mathrm{mmol} / \mathrm{L}$, serum creatinine: $1.1 \mathrm{mg} / \mathrm{dl}$, serum urea: $12 \mathrm{mmol} / \mathrm{l}$, serum bicarbonate: $22 \mathrm{mmol} / \mathrm{l}$, serum glucose: $10 \mathrm{mmol} / \mathrm{l}$, white blood cells: $3.5 \mu \mathrm{l}$, hemoglobin: $13.7 \mathrm{~g} / \mathrm{dL}$, platelets: 420,000, aspartate transaminase: $24 \mathrm{U} / \mathrm{L}$, alanine aminotransferase: $16 \mathrm{U} / \mathrm{L}$, albumin: $36 \mathrm{~g} / \mathrm{L}$. The patient was admitted with a diagnosis of TEN into the dermatology ward of Afzalipour hospital, of Kerman University of Medical Sciences, Iran that also is a referral COVID-19 center hospital in Kerman. Lamotrigine was stopped and he was managed with supportive care, wound care, thrombotic prophylaxis. As corticosteroid is rather controversial during covid-19 outbreak and IVIG which is the first choice of treatment was not afforded for the patient, we worked up to start cyclosporine. The blood pressure, electrolytes and cholesterol were normal. Therefore, we started cyclosporine $4 \mathrm{mg} / \mathrm{kg} /$ day. Within the first 36 hours after starting cyclosporine, the patient showed a dramatic response to the treatment. No new bullae formation was detected and there was a reduction in erythema and erosion. The evidence of re-epithelization was observed on the third day and the Nikolsky sign became negative (Figure 2). Meanwhile, blood pressure and kidneys' function were normal. Eventually, he discharged after one week with prescription of the cyclosporin $2 \mathrm{mg} / \mathrm{kg} /$ day for another one week. He informed respecting the possibility of relapse. One week later the patient referred to our clinic. Skin and mucosal lesions improved and the cyclosporine therapy was discontinued.Discussion: Although systemic corticosteroid, IVIG, cyclosporine, and TNF inhibitors are optional drugs in managing TEN, it is still controversial about the gold standard treatment for TEN ${ }^{[1,4]}$. During COVID-19 outbreak, the management of TEN has encountered an additive challenge. Because use of the immunosuppressive drug, especially like prednisolone and cyclosporine, are controversial ${ }^{[8]}$. It seems IVIG is the best option for the management of TEN, because IVIG is human immunoglobulin and can strengthens the immune system [9]. An important issue in patients that IVIG is not affordable, as it is the case in our patient, or is contraindicated, is which one of immunosuppressive drugs can be use in the management of TEN during covid-19 outbreak. As we know, there is not any study in COVID-19 outbreak to compare the effects of different immunosuppressive drugs such as cyclosporine and prednisolone on TEN patients. Use of corticosteroid, which is the routine choice in treating TEN patients, is controversial in COVID-19 outbreak especially amongst critical patients, ${ }^{[10,11]}$. Albeit, it appears that corticosteroids can be effective in severe COVID-19 cases, its routine use is not supported in the literature ${ }^{[12-14]}$. On the other hand, due to the increasing mortality of seasonal epidemic of influenza among corticosteroid users, administrating corticosteroids in TEN patients would be of concern ${ }^{[15]}$. Therefore, we discarded the use of corticosteroid. We decided to start cyclosporine, as it can be a good treatment option in COVID-19 outbreak due to several reasons. First, in Kirchhof et al. ${ }^{[16]}$ cohort study the relative mortality of TEN patients who were treated with cyclosporine was lower than those with IVIg. Second, cyclosporine might be associated with a rapid re-epithelialization ${ }^{[17]}$ and reduce duration of hospitalization ${ }^{[17,18]}$, which can reduce the risk of COVID19 in critical patients during hospitalization. Third, cyclosporine can inhibit influenza A virus ${ }^{[19]}$. forth, in-vitro studies showed that cyclosporine can inhibit immunophilin pathway and by this way it can inhibit replication of coronavirus ${ }^{[8,19-22]}$. Fifth, COVID-19 mortality is highly linked to the cytokine storm and cyclosporine can be beneficial during the inflammatory phase of COVID-19 ${ }^{[21,22]}$. Sixth, Cavagana et al. ${ }^{[22]}$ reported that clinical course of COVID-19 patients on calcineurin inhibitors (CNIs) is generally mild with a low risk of superinfection. Finally, although cyclosporine is an immunosuppressant agent, infections are not one of its common side effects ${ }^{[23]}$. Conclusion: IVIG is the best treatment option in the management of TEN during COVID-19 outbreak. For those IVIG is contraindicated or is not affordable, cyclosporine can be a better choice than other immunosuppressant drugs. As, patients on cyclosporine can benefit its antiviral effects and have a shorter hospital admission stay.Potential conflicts of interest: The authors declare no conflicts of interest.References:

1. Kinoshita, Y., \& Saeki, H. (2017). A review of toxic epidermal necrolysis management in Japan. Allergology international : official journal of the Japanese Society of Allergology, 66(1), 36-41.

2. Rijal, J. P., Pompa, T., Giri, S., \& Bhatt, V. R. (2014). A case of toxic epidermal necrolysis caused 
by trimethoprim-sulfamethoxazole. BMJ case reports, 2014, bcr2013203163.

3. Mukasa, Y., \& Craven, N. (2008). Management of toxic epidermal necrolysis and related syndromes. Postgraduate medical journal, 84(988), 60-65.

4. Hasegawa, A., \& Abe, R. (2020). Recent advances in managing and understanding Stevens-Johnson syndrome and toxic epidermal necrolysis. F1000Research, 9, F1000 Faculty Rev-612.

5. Harr, T., \& French, L. E. (2010). Toxic epidermal necrolysis and Stevens-Johnson syndrome. Orphanet journal of rare diseases, 5,39 .

6. Charlton, O. A., Harris, V., Phan, K., Mewton, E., Jackson, C., \& Cooper, A. (2020). Toxic Epidermal Necrolysis and Steven-Johnson Syndrome: A Comprehensive Review. Advances in wound care, 9(7), 426-439.

7. Schoot, T. S., Kerckhoffs, A., Hilbrands, L. B., \& van Marum, R. J. (2020). Immunosuppressive Drugs and COVID-19: A Review. Frontiers in pharmacology, 11, 1333.

8. Poulsen, N. N., von Brunn, A., Hornum, M., \& Blomberg Jensen, M. (2020). Cyclosporine and COVID19: Risk or favorable?. American journal of transplantation: official journal of the American Society of Transplantation and the American Society of Transplant Surgeons, 20(11), 2975-2982.

9. Dhar S. (2009). Intravenous immunoglobulin in dermatology. Indian journal of dermatology, 54(1), $77-79$.

10. Li, H., Chen, C., Hu, F., Wang, J., Zhao, Q., Gale, R. P., \& Liang, Y. (2020). Impact of corticosteroid therapy on outcomes of persons with SARS-CoV-2, SARS-CoV, or MERS-CoV infection: a systematic review and meta-analysis. Leukemia, 34(6), 1503-1511.

11. RECOVERY Collaborative Group, Horby, P., Lim, W. S., Emberson, J. R., Mafham, M., Bell, J. L., Linsell, L., Staplin, N., Brightling, C., Ustianowski, A., Elmahi, E., Prudon, B., Green, C., Felton, T., Chadwick, D., Rege, K., Fegan, C., Chappell, L. C., Faust, S. N., Jaki, T., .. Landray, M. J. (2020). Dexamethasone in Hospitalized Patients with Covid-19 - Preliminary Report. The New England journal of medicine, NEJMoa2021436. Advance online publication.

12. Yang, Z., Liu, J., Zhou, Y., Zhao, X., Zhao, Q., \& Liu, J. (2020). The effect of corticosteroid treatment on patients with coronavirus infection: a systematic review and meta-analysis. The Journal of infection, 81(1), e13-e20.

13. Veronese N, Demurtas J, Yang L, Tonelli R, Barbagallo M, Lopalco P, Lagolio E, Celotto S, Pizzol D, Zou L, Tully MA, Ilie PC, Trott M, López-Sánchez GF, Smith L. Use of Corticosteroids in Coronavirus Disease 2019 Pneumonia: A Systematic Review of the Literature. Front Med (Lausanne). 2020 Apr $24 ; 7: 170$.

14. Russell, C. D., Millar, J. E., \& Baillie, J. K. (2020). Clinical evidence does not support corticosteroid treatment for 2019-nCoV lung injury. Lancet (London, England), 395(10223), 473-475.

15. Abdollahimajd, F., Shahidi-Dadras, M., M Robati, R., \& Dadkhahfar, S. (2020). Management of Pemphigus in COVID-19 Pandemic Era; a Review Article. Archives of academic emergency medicine, 8(1), e51.

16. Kirchhof, M. G., Miliszewski, M. A., Sikora, S., Papp, A., \& Dutz, J. P. (2014). Retrospective review of Stevens-Johnson syndrome/toxic epidermal necrolysis treatment comparing intravenous immunoglobulin with cyclosporine. Journal of the American Academy of Dermatology, 71(5), 941-947.

17. Arévalo, J. M., Lorente, J. A., González-Herrada, C., \& Jiménez-Reyes, J. (2000). Treatment of toxic epidermal necrolysis with cyclosporin A. The Journal of trauma, 48(3), 473-478.

18. Kumar, R., Das, A., \& Das, S. (2018). Management of Stevens-Johnson Syndrome-Toxic Epidermal Necrolysis: Looking Beyond Guidelines!. Indian journal of dermatology, 63(2), 117-124.

19. Rudnicka, L., Glowacka, P., Goldust, M., Sikora, M., Sar-Pomian, M., Rakowska, A., Samochocki, Z., \& Olszewska, M. (2020). Cyclosporine therapy during the COVID-19 pandemic. Journal of the American Academy of Dermatology, 83(2), e151-e152.

20. Gelincik, A., Brockow, K., Çelik, G. E., Doña, I., Mayorga, C., Romano, A., Soyer, Ö., AtanaskovicMarkovic, M., Barbaud, A., \& Torres, M. J. (2020). Diagnosis and management of the drug hypersensitivity reactions in Coronavirus disease 19: An EAACI Position Paper. Allergy, 75(11), 2775-2793.

21. Rodriguez-Cubillo, B., de la Higuera, M., Lucena, R., Franci, E. V., Hurtado, M., Romero, N. C., More- 
no, A. R., Valencia, D., Velo, M., Fornie, I. S., \& Sanchez-Fructuoso, A. I. (2020). Should cyclosporine be useful in renal transplant recipients affected by SARS-CoV-2?. American journal of transplantation: official journal of the American Society of Transplantation and the American Society of Transplant Surgeons, 20(11), 3173-3181.

22. Cavagna, L., Seminari, E., Zanframundo, G., Gregorini, M., Di Matteo, A., Rampino, T., Montecucco, C., Pelenghi, S., Cattadori, B., Pattonieri, E. F., Vitulo, P., Bertani, A., Sambataro, G., Vancheri, C., Biglia, A., Bozzalla-Cassione, E., Bonetto, V., Monti, M. C., Ticozzelli, E., Turco, A., .. Meloni, F. (2020). Calcineurin Inhibitor-Based Immunosuppression and COVID-19: Results from a Multidisciplinary Cohort of Patients in Northern Italy. Microorganisms, 8(7), 977.

23. Colombo, D., Chimenti, S., Grossi, P., Marchesoni, A., Di Nuzzo, S., Griseta, V., Gargiulo, A., Parodi, A., Simoni, L., \& Bellia, G. (2014). Prevalence of past and reactivated viral infections and efficacy of cyclosporine A as monotherapy or in combination in patients with psoriatic arthritis-synergy study: a longitudinal observational study. BioMed research international, 2014, 941767.

Figure legends:Figure 1: On arrival skin lesions Figure 2: Improved skin lesions at day 4 of admission after starting cyclosporine 


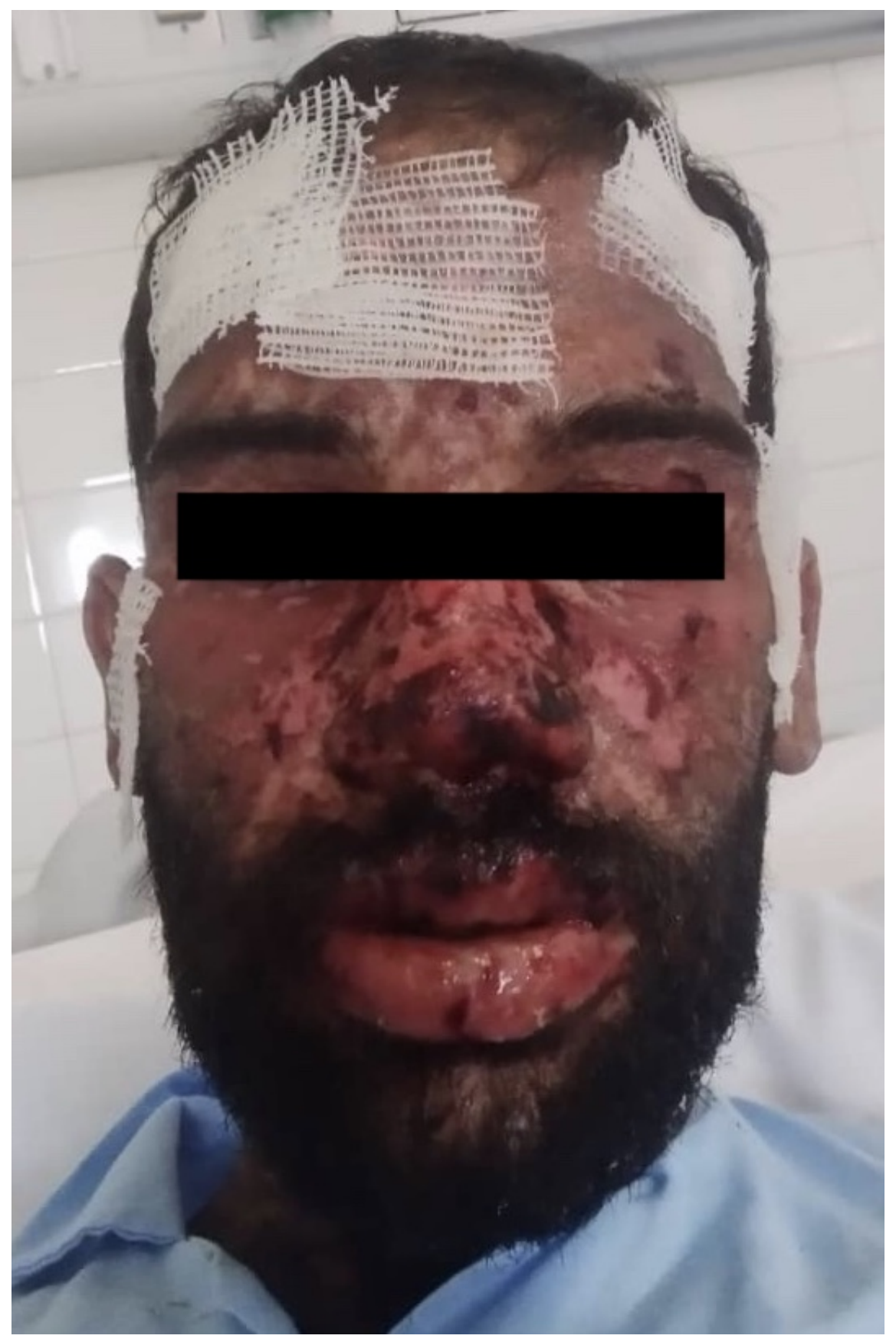




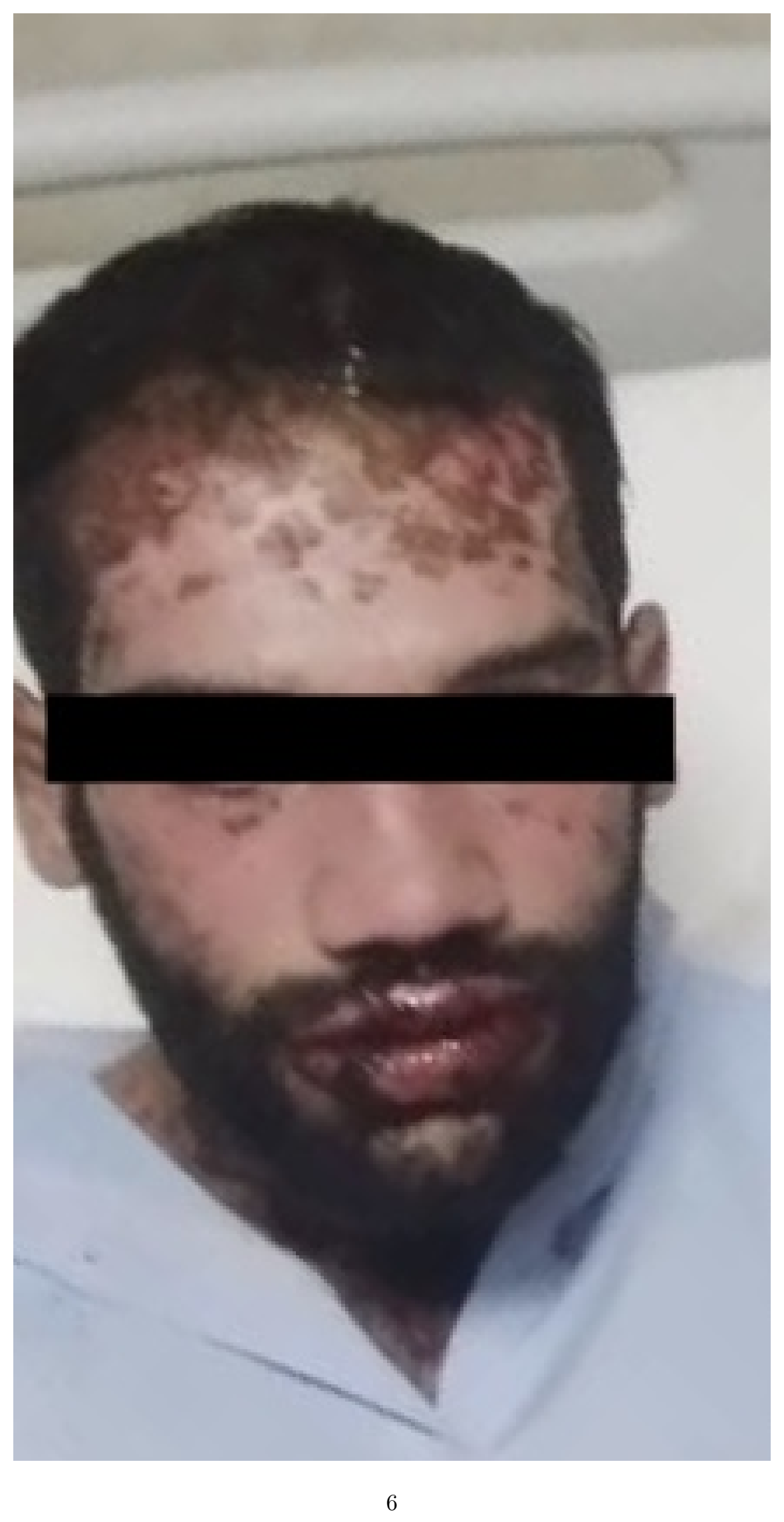

